\title{
Theoretical Study on the Effects of Dislocations in Monolithic III-V Lasers on Silicon
}

\author{
Constanze Hantschmann, Student Member, IEEE, Zizhuo Liu, Student Member, IEEE, Mingchu Tang, \\ Member, IEEE, Siming Chen, Member, IEEE, Alwyn J. Seeds, Fellow, IEEE, Huiyun Liu, Senior \\ Member, IEEE, Ian H. White, Fellow, IEEE, and Richard V. Penty, Senior Member, IEEE
}

\begin{abstract}
In this work, we present an approach to modelling III-V lasers on silicon based on a travelling-wave rate equation model with sub-micrometer resolution. By allowing spatially resolved inclusion of individual dislocations along the laser cavity, our simulation results offer new insights into the physical mechanisms behind the characteristics of $980 \mathrm{~nm} \operatorname{In}(\mathrm{Ga}) \mathrm{As} / \mathrm{GaAs}$ quantum well $(\mathrm{QW})$ and $1.3 \mu \mathrm{m}$ quantum $\operatorname{dot}(\mathrm{Q} D)$ lasers grown on silicon. By studying the reduction of the local gain in carrierdepleted regions around dislocation locations and the resulting impact on threshold current increase and slope efficiency at high dislocation densities, we identify two effects with particular importance for practical applications. First, a large minority carrier diffusion length is a key parameter inhibiting laser operation by enabling carrier migration into dislocations over larger areas, and secondly, increased gain in dislocation-free regions compensating for gain dips around dislocations may contribute to gain compression effects observed in directly modulated silicon-based QD lasers. We believe that this work is an important contribution in creating a better understanding of the processes limiting the capabilities of III-V lasers on silicon in order to explore suitable materials and designs for monolithic light sources for silicon photonics.
\end{abstract}

Index Terms-Quantum dot lasers, quantum well lasers, semiconductor device modeling, silicon photonics

\section{INTRODUCTION}

$\mathrm{D}$ ISLOCATION-INDUCED performance degradation with respect to luminescence efficiency and reliability has been a major concern for semiconductor researchers ever since the early days of semiconductor injection lasers in the 1970s [1][3]. Modern epitaxial growth techniques have matured to a level that large-area lattice-matched multi-layer wafers with dislocation densities smaller than $10^{4} \mathrm{~cm}^{-2}$ can be produced [4], meaning that statistically nine out of ten $2 \times 500 \mu^{2}$ lasers have an entirely dislocation-free active region. As a consequence, the focus of the latest research has shifted towards realizing more challenging structures, with the epitaxial growth of III-Vs on silicon gaining particular attention due to the prospect of merging high optical gain materials with silicon photonics. The high density of threading dislocations emerging from the lattice-mismatched III-V/silicon interface and propagating up

Manuscript received December 23 2019. This work is supported in part by UK EPSRC. C. Hantschmann's Ph.D. studies are funded by Qualcomm Inc.

C. Hantschmann, I. H. White, and R. V. Penty are with the Centre for Photonic Systems, Department of Engineering, University of Cambridge, Cambridge CB3 0FA, UK. (e-mail: cb893@cam.ac.uk, ihw3@cam.ac.uk, rvp11@cam.ac.uk). into the active region remains, however, the main factor limiting the progress of incorporating III-V-on-Si gain blocks in monolithically integrated silicon photonic optoelectronic circuits. Despite considerable advances in crystal growth technology and the optimization of defect filter and buffer layers, quantum well (QW) lasers on silicon have failed to work reliably [5]-[8]. Instead, InAs/GaAs quantum dots (QDs) have established themselves as the gain medium of choice for mismatched growth due to their higher tolerance to dislocations, since ultrafast carrier capture into QDs reduces the effective diffusion length and prevents the majority of injected carriers from migrating into defects [9]-[11].

To date, the performance differences seen for QD and QW lasers grown on silicon remain relatively unexplored. In our previous work, we have shown travelling-wave rate equation simulations using a macroscopically averaged carrier loss time inferred from a dislocation-limited diffusion length in order to explain the performance discrepancies between GaAs-based QW and QD lasers grown on silicon [9]. While this model was capable of reproducing experimentally observed performance trends, it allowed only limited access to the underlying physical processes. Here, we introduce therefore an approach to simulating such devices by using a rate equation travelling-wave model with sub-micrometer spatial resolution, which enables the inclusion of individual dislocations in the laser cavity. The objective of this work is to identify the main mechanisms responsible for the drop in performance typically observed at dislocation densities $\geq 10^{6} \mathrm{~cm}^{-2}$, with a special focus on the limitations QW structures experience. Our highresolution approach points out the role diffusion plays in the functionality of QW and QD devices and gives new insights into the local phenomena arising from the interplay of gain, the carrier density, and the photon density in the presence of dislocations

\section{EXPERIMENTAL PERFORMANCE TRENDS}

The performance dependence of III-V lasers grown on silicon on dislocation density offers a route to understanding the fundamental implications dislocations have each for QD and QW devices. Fig. 1 suggests that there is a correlation between

Z. Liu, M. Tang, S. Chen, A. J. Seeds, and H. Y. Liu are with the Department of Electronic and Electrical Engineering, University College London, London WC1E 7JE, UK. (e-mail: zizhuo.liu.16@ucl.ac.uk, mingchu.tang.11@ucl.ac.uk, siming.chen@ucl.ac.uk, a.seeds@ucl.ac.uk, huiyun.liu@ucl.ac.uk).

I. H. White is also with the University of Bath, Bath BA2 7AY, UK. 


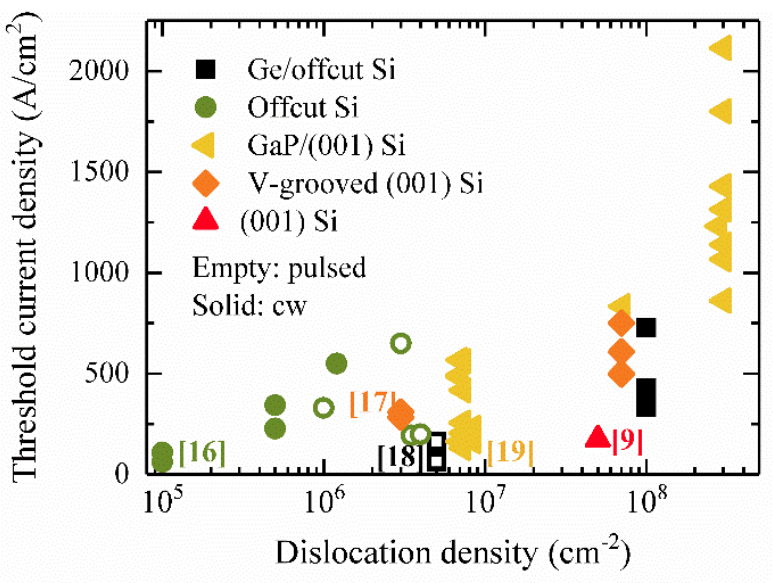

Fig. 1. Threshold current density against dislocation density of $1.3 \mu \mathrm{m}$ InAs/GaAs QD lasers by substrate type [9], [16]-[19]. Results are shown for conventional Fabry-Pérot-type devices operating at room temperature from publications with stated dislocation density.

high threshold current densities and high dislocation densities in monolithic QD lasers on silicon. This is consistent with the perception that dislocations act as nonradiative defect centers [1], [3], [12], [13], which result in higher laser thresholds through increased carrier loss [14]. More data points at low dislocation densities would be necessary though to pinpoint a trend with greater confidence. Whereas good lasing characteristic have been obtained even for dislocation densities as high as $3 \times 10^{8} \mathrm{~cm}^{-2}$ [15], monolithic GaAs-based QW lasers on silicon have only been reported in the comparably small dislocation range of $2 \times 10^{6} \mathrm{~cm}^{-2}$ to $10^{7} \mathrm{~cm}^{-2}$ [8], [20]-[23]. Together with the lack of systematic studies comparing similar devices at varying dislocation densities, this makes these data insufficient for the identification of reliable performance trends. Bearing in mind that QD lasers in general can intrinsically have lower threshold currents due to their reduced active region volume, the many publications reporting QW laser threshold current densities of several $\mathrm{kA} / \mathrm{cm}^{2}$ [21], [23]-[25], as opposed to the few published sub- $\mathrm{kA} / \mathrm{cm}^{2}$ thresholds [23], [26]-[28], suggest nevertheless that QW lasers are more susceptible to dislocation-induced threshold current increase.

Although the literature is much less clear with respect to a possible light-current $(L I)$ slope dependence due to the influence of the laser cavity and active region design, the authors of [29] and [30] report a distinct slope efficiency drop with rising dislocation number. In [30] it is suggested that the slope reduction may be a consequence of increased optical scattering loss at dislocation cores or of a reduced internal quantum efficiency. Based on the available data in the literature, there is no clear indication as to what extent the same applies to QW lasers.

\section{NuMERICAL MODEL}

A standard multi-level rate equation travelling-wave model is used to simulate narrow-ridge waveguide QD and QW lasers grown on silicon substrate [31]-[34]. The carrier equations (1) and (2) for computation of the QW carrier numbers comprise
TABLE I

OVERVIEW OF THE USED SIMULATION PARAMETERS AND VARIABLES. COMMON PARAMETERS FOR QW AND QD SIMULATIONS

Spatial step size $\Delta z=500 \mathrm{~nm} \quad$ Dislocation capture time $\tau_{\text {dis }}=10 \mathrm{ps}$

Cavity length $L=500 \mu \mathrm{m} \quad$ BL, QW/WL diffusion length $L_{\text {diff }}=$

Waveguide width $w=2 \mu \mathrm{m}$

Facet reflectivity $R_{l, 2}=0.95,0.30$

Number of active layers $=5$

Optical loss $\alpha_{i}=5 \mathrm{~cm}^{-1}$

Injection efficiency $\eta=0.55$

$$
10 \mu \mathrm{m}
$$

Nonradiative lifetime $\tau_{n r}=1 \mathrm{~ns}$ (all levels)

$\mathrm{QW} / \mathrm{WL}$ escape time $\tau_{\text {esc }}^{Q W, W L}=2.2 \mathrm{ps}$

QD PARAMETERS

\begin{tabular}{lc}
\hline \hline Wavelength $\lambda=1300 \mathrm{~nm}$ & GS, ES escape time $\tau_{e s c}^{G S, E S}=2.7 \mathrm{ps}$, \\
QD density $\rho=6 \times 10^{10} \mathrm{~cm}^{-2}$ & $34 \mathrm{ps}$ \\
Modal gain $g_{\text {mod }}=50 \mathrm{~cm}^{-1}$ & SCH region transport and WL \\
Confinement factor $\Gamma=0.005$ & capture time $\tau_{\text {cap }}^{W L}=3.4 \mathrm{ps}$ \\
SCH region thickness $=250 \mathrm{~nm}$ & QD carrier capture time $\tau_{c a p}^{Q D}=3 \mathrm{ps}$ \\
WL thickness $=8 \mathrm{~nm}$ & QD relaxation time $\tau_{\text {relax }}^{Q D}=0.5 \mathrm{ps}$ \\
Gain compression factor $\varepsilon=$ & Ground state hole occupation \\
$1 \times 10^{-16} \mathrm{~cm}^{3}$ & probability $f_{G S}^{h}=0.5$ \\
& QW PARAMETERS \\
\hline \hline Wavelength $\lambda=980 \mathrm{~nm}$ & SCH region transport and QW capture \\
Gain constant $g_{0}=3000 \mathrm{~cm}^{-1}$ & time $\tau_{\text {cap }}^{Q W}=3 \mathrm{ps}$ \\
Confinement factor $\Gamma=0.05$ & SCH region thickness $=65 \mathrm{~nm}$ \\
Gain compression factor $\varepsilon=$ & QW thickness $=7.5 \mathrm{~nm}$ \\
$1 \times 10^{-17} \mathrm{~cm}^{3}$ & Transparency carrier density $n_{t r}=$ \\
& $1 \times 10^{18}$ cm ${ }^{-3}$ \\
\hline \hline Olementary charge $e$ & QW, QD volume $V_{Q W}, V_{Q D}$ \\
Group velocity $v_{g r}$ & Spontaneous noise $i_{s p}$ \\
Wavelength detuning $\delta$ & QD maximum material gain $g_{\text {mat }}^{\text {max }}=$ \\
Diffusion time constant $\tau_{\text {diff }}=$ & $g_{\text {mod }} / \Gamma$ \\
$\Delta z^{2} /(2 D)$ with $L_{\text {diff }}=\sqrt{D \tau_{n r}}$ & \\
\hline \hline
\end{tabular}

two levels representing the QWs themselves and the barrier layers (BLs) including current injection, carrier capture, relaxation, and re-emission, nonradiative recombination and dislocation-induced carrier loss, as well as a lateral diffusion term and lasing. It should be noted that $\tau_{\text {dis }}$ is a vector inducing carrier loss at predefined dislocation positions only rather than being an effective laser parameter, as further explained in Section III. An overview of all used variables and parameters can be found in Table I.

$$
\begin{aligned}
& \frac{d N_{B L}}{d t}=\frac{\eta I}{e} \frac{\Delta z}{L}-\frac{N_{B L}}{\tau_{c a p}^{Q W}}+\frac{N_{Q W}}{\tau_{e s c}^{Q W}}-\frac{N_{B L}}{\tau_{n r}}-\frac{N_{B L}}{\tau_{d i s}}-\frac{\Delta N_{B L}}{\tau_{d i f f}} \\
& \frac{d N_{Q W}}{d t}=\frac{N_{B L}}{\tau_{c a p}^{Q W}}-\frac{N_{Q W}}{\tau_{e s c}^{Q W}}-\frac{N_{Q W}}{\tau_{n r}}-\frac{N_{Q W}}{\tau_{\text {dis }}}-\frac{\Delta N_{Q W}}{\tau_{\text {diff }}}-v_{g r} g_{Q W} S \frac{\Delta z V_{Q W}}{L}
\end{aligned}
$$

The QD carrier rate equations contain two additional levels representing the QD ground and the first excited state. The main distinction of (4) - (6) is the inclusion of the QD occupation factors $f_{G S, E S}^{\prime}=\left(1-f_{E S, G S}\right)$ in the QD levels, and the fact that carrier migration is only possible in the $\mathrm{BL}$ and wetting layer (WL) due to the in-plane carrier confinement given by the spatially isolated QDs [11]. For our purposes, an excitonic approach is deemed sufficient, since electrons have a higher likelihood of interacting with dislocations due to their higher mobility compared with holes. Migration of electrons into dislocations is, therefore, expected to pose the limiting factor 
for the performance of bipolar devices on silicon [35].

$\frac{d N_{B L}}{d t}=\frac{\eta I}{e} \frac{\Delta z}{L}-\frac{N_{B L}}{\tau_{c a p}^{W L}}+\frac{N_{W L}}{\tau_{e s c}^{W L}}-\frac{N_{B L}}{\tau_{n r}}-\frac{N_{B L}}{\tau_{d i s}}-\frac{\Delta N_{B L}}{\tau_{\text {diff }}}$

$\frac{d N_{W L}}{d t}=\frac{N_{B L}}{\tau_{c a p}^{W L}}-\frac{N_{W L}}{\tau_{e s C}^{W L}}+\frac{N_{E S}}{\tau_{e s C}^{E S}}-\frac{N_{W L} f_{E S}^{\prime}}{\tau_{c a p}^{Q D}}-\frac{N_{W L}}{\tau_{n r}}-\frac{N_{W L}}{\tau_{\text {dis }}}-\frac{\Delta N_{W L}}{\tau_{\text {diff }}}$

$\frac{d N_{E S}}{d t}=\frac{N_{W L} f_{E S}^{\prime}}{\tau_{c a p}^{Q D}}-\frac{N_{E S}}{\tau_{e S C}^{E S}}+\frac{N_{G S} f_{E S}^{\prime}}{\tau_{e S C}^{G S}}-\frac{N_{E S v} f_{G S}^{\prime}}{\tau_{\text {relax }}^{Q D}}-\frac{N_{E S}}{\tau_{n r}}$

$\frac{d N_{G S}}{d t}=\frac{N_{E S} f_{G S}^{\prime}}{\tau_{\text {relax }}^{Q D}}-\frac{N_{G S} f_{E S}^{\prime}}{\tau_{e S C}^{G S}}-\frac{N_{G S}}{\tau_{n r}}-v_{g r} g_{Q D} S \frac{\Delta z V_{Q D}}{L}$

The electronic properties given by the carrier equations couple with the laser's optical behavior via the QW and QD gain functions (7) and (8) and the photon density $S$, which is computed based on the forward and reverse travelling-wave electric field $E^{ \pm}$equations (9). A digital gain filter is applied to (9) in order to shape the gain spectrum acting on the optical fields [36]. The QW model is set up for emission at $980 \mathrm{~nm}$, while the QD model lases at $1.3 \mu \mathrm{m}$. The different emission wavelengths reflect practical device epistructures and do not affect the simulation results shown in the following figures.

$$
\begin{gathered}
g_{Q W}=g_{0} \ln \left({ }^{N_{Q W}} / N_{t r}\right) /(1+\varepsilon S) \\
g_{Q D}=g_{m a t}^{\max }\left(f_{G S}^{e}+f_{G S}^{h}-1\right) /(1+\varepsilon S) \\
\left(\frac{1}{v_{g r}} \frac{\partial}{\partial t} \pm \frac{\partial}{\partial z}\right) E^{ \pm}(z, t)=\left(\frac{\Gamma g}{2}-\frac{\alpha_{i}}{2}-i \delta\right) E^{ \pm}(z, t)+i_{s p}^{ \pm}(z, t)
\end{gathered}
$$

Many travelling-wave models are suitable for relatively large space steps $\Delta z$ of around $10 \mu \mathrm{m}$, since the maximum possible $\Delta z$ is dictated by the smallest deployed time constants via $\Delta z=v_{g r} \Delta t$. Although large $\Delta z$ are advantageous in terms of simulation time, they only allow the use of averaged, effective laser parameters, for instance an overall reduced nonradiative recombination time to account for an increased number of dislocations in the active region, which are not entirely able to describe the physics of III-V lasers on silicon accurately. In these simulations, $\Delta z$ is, therefore, reduced to the submicrometer level, where a step size of $500 \mathrm{~nm}$ offers a good trade-off between physical accuracy and computational feasibility. The Courant Friedrichs Lewy condition is equal to 1, ensuring numerical stability [37].

\section{IMPLEMENTATION OF DISLOCATIONS}

In order to study the effects of threading dislocations in GaAs-based laser active regions, nonradiative recombination centers are placed on a regular grid along the laser cavity, modelled as laser sections of ultrafast carrier loss at rate $\tau_{d i s}{ }^{-1}$ in order to represent fast carrier capture into defect states. Since the exact dislocation carrier capture time is not known, we assume here a capture time of the order of a few picoseconds, comparable with the one into QD states [38], [39]. In reality, the physical radius of an actual dislocation core is estimated to be only a few tens of nanometers [40], [41], so varying $\tau_{\text {dis }}$ is not only a means of modelling realistic performance trends with growing dislocation density, but also a way to compensate for larger $\Delta z$, which overestimate the dislocation size slightly. For $\Delta z=500 \mathrm{~nm}$, a value of $\tau_{d i s}=10 \mathrm{ps}$ is chosen to model dislocation sections, whereas $\tau_{\text {dis }}$ is set to infinity in dislocation-free regions.

It should be noted that a series of assumptions and simplifications is made in the present approach. First, all simulations are performed for laser cavities no wider than $2 \mu \mathrm{m}$. This is not only to ensure single transverse mode operation, but also to be able to neglect radial carrier diffusion into dislocations, which technically requires a two-dimensional simulation approach. Secondly, while a regular arrangement of dislocations in the active region is best for the reproducibility of simulation results, it tends to be the worst-case scenario in terms of laser performance [42], as the number of carriers affected by diffusion-assisted carrier loss is maximized. A (a)

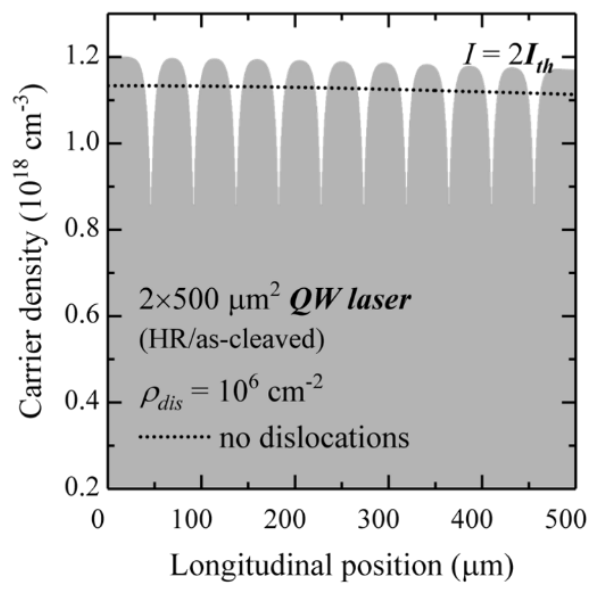

(b)

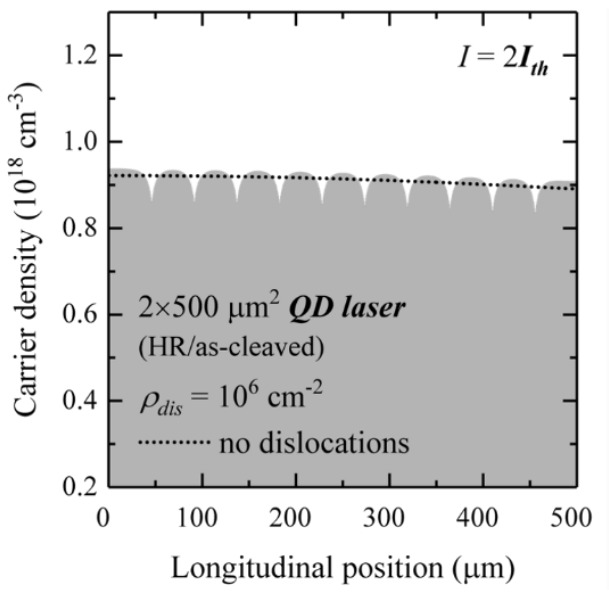

(c)

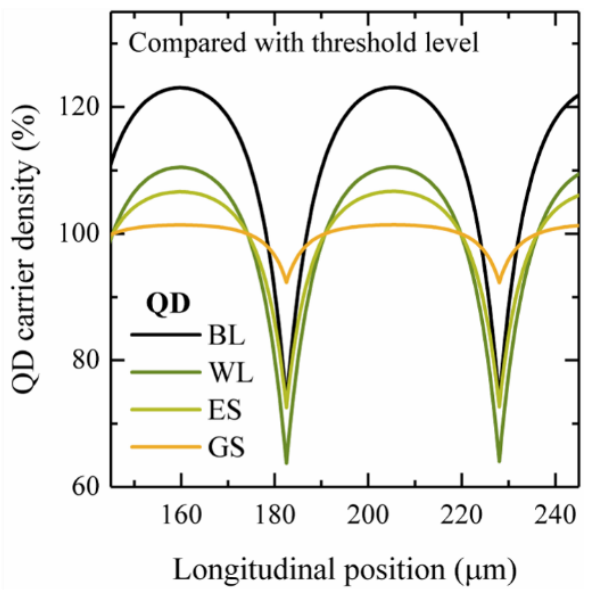

Fig. 2. (a) QW and (b) QD ground state carrier density of $2 \times 500 \mu \mathrm{m}^{2}$ high-reflection (HR) coated/as-cleaved lasers at $\sim 2 I_{t h}$ against the longitudinal position in the presence of ten dislocations in comparison with the carrier density level without dislocations at the same optical output power level (dotted line). For the QD active region, the effect of dislocations propagating through the BL and WL into the dots is neglected due to the typically very small percentage of affected dots. (c) Carrier density in the QD BL, WL, excited state, and ground state (at the same current as Fig. 2 (b)) against the longitudinal position in relation to the dislocation-free threshold level. 
realistic III-V laser grown on silicon has a random dislocation distribution allowing for variations in the laser performance, where slightly better than average performance is obtained from devices with large dislocation-free regions. Lastly, dislocationinduced carrier loss is ignored in the QD levels due to the negligible number of threading dislocations compared with the number of QDs per active layers.

\section{KeY FINDINGS AND DisCUSSION}

Fig. 2(a) shows an example of the QW carrier density distribution at twice the laser threshold obtained for a dislocation density $\rho_{\text {dis }}$ of $1 \times 10^{6} \mathrm{~cm}^{-2}$, equating to ten dislocations in a $2 \times 500 \mu \mathrm{m}^{2}$ laser, as well as the carrier density distribution at the same output power level in the absence of dislocations. It can clearly be seen that the influence of carrier loss at the only $500 \mathrm{~nm}$-long dislocation positions is intensified by carrier diffusion, which causes the carrier density over several micrometers to fall below the threshold value. The large minority carrier diffusion length in GaAs-based III-V materials, set to $10 \mu \mathrm{m}$ in these simulations, contributes therefore to an increased sensitivity to defects [35]. The carrier density in dislocation-free regions must, therefore, be increased in order to attain the required cavity threshold gain. The QD carrier profile depicted in Fig. 2(b), on the other hand, illustrates that ultrafast carrier capture into the dot states prevents strong dips in the ground state carrier density. As a consequence, the ground state carrier occupation in the vicinity of dislocations drops by only about $10 \%$, despite the BL and WL carrier densities dropping to about $70 \%$ of their dislocation-free threshold level (Fig. 2(c)). The QD excited state carrier density is similarly reduced, but only since captured carriers relax directly into the ground state.

The behaviors shown in Figs. 2(a) and (b) are reflected in the local gain profiles as depicted in Figs. 3(a) and (b). Fig. 3(a) reveals that carrier-depleted areas around dislocations in the QWs are highly absorptive due to the logarithmic nature of the QW gain function. It is only at higher current injection levels that defect states become populated, as indicated in dark grey, so that diffusion-assisted carrier loss becomes less severe [43], (a)

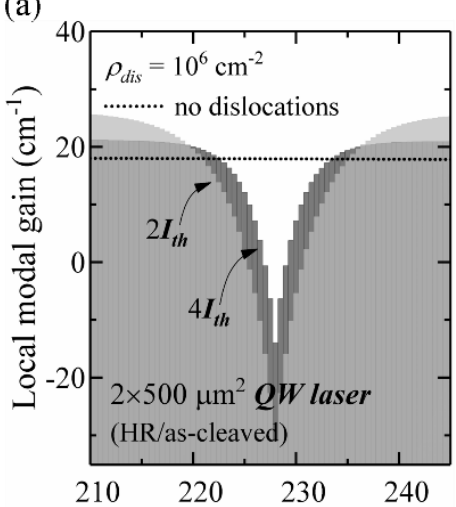

(b)

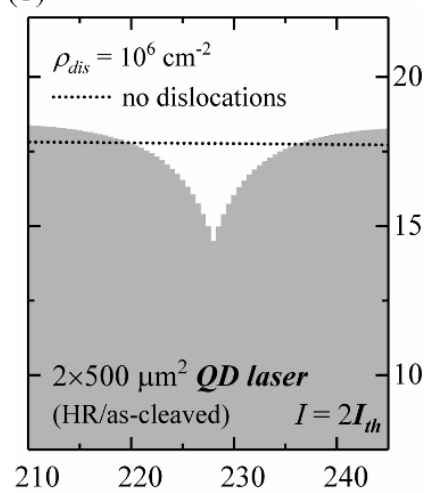

Longitudinal position $(\mu \mathrm{m})$ Longitudinal position $(\mu \mathrm{m})$

Fig. 3. Insets of the (a) QW and (b) QD gain profile around a dislocation in comparison with the gain profile without dislocations at the same power level (dotted line). The effect of the $10 \mu \mathrm{m}$-long diffusion length can clearly be seen. Fig. 3(a) illustrates the saturation of defect states with rising injection level $\left(\sim 2 I_{t h}\right.$ in light grey, $\sim 4 I_{t h}$ at dark grey). The gain dips in Fig. 3(b) result from locally reduced BL and WL carrier densities, as shown in Fig. 2(c). Note the different $y$ axis scales for the QD and QW gain.

[44]. The QD modal gain, in contrast, exhibits only a minor dip in the vicinity of a dislocation, as the high ground state occupation due to fast carrier capture helps to ensure overall high gain. It is interesting to note that the threshold gain in individual regions moves closer to the maximum ground state gain of $25 \mathrm{~cm}^{-1}$ (after (8), assuming maximum electron and hole ground state occupation probabilities of 1 and 0.5 , respectively), as this increases gain compression effects [45]. This is potentially a smaller issue for high-gain QD lasers as modelled here, but might pose a problem for QD lasers at high dislocation densities with limited modal gain $\left(10 \mathrm{~cm}^{-1}\right.$ $20 \mathrm{~cm}^{-1}$, for example). Besides high damping induced by a short differential carrier lifetime, this offers another possible explanation of the large $K$-factors so far observed in directly modulated QD lasers grown on silicon [46], [47].

Figs. 4(a) and (b) show results of simulated QD and QW $L I$ curves for device dimensions of $2 \times 500 \mu \mathrm{m}^{2}$ at different $\rho_{\text {dis }}$ confirming the experimentally observed trends of increased (a)

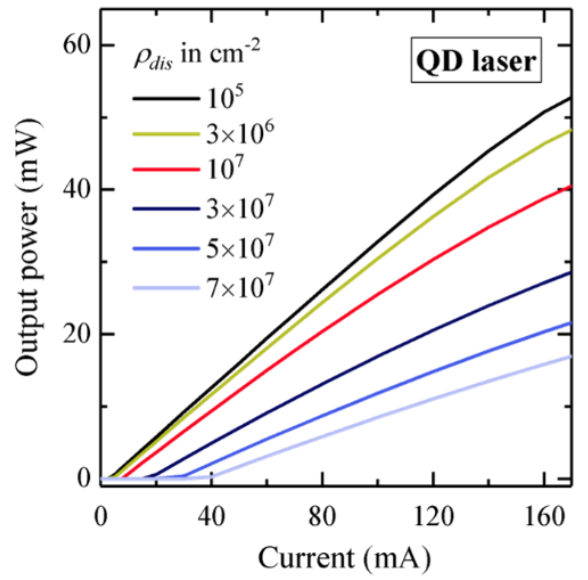

(b)

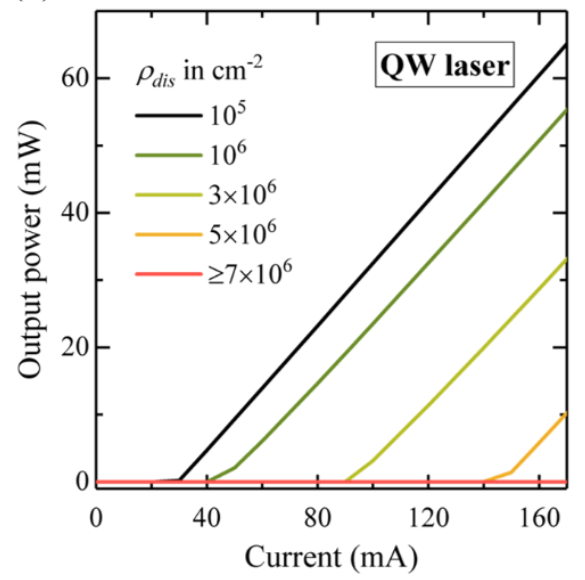

(c)

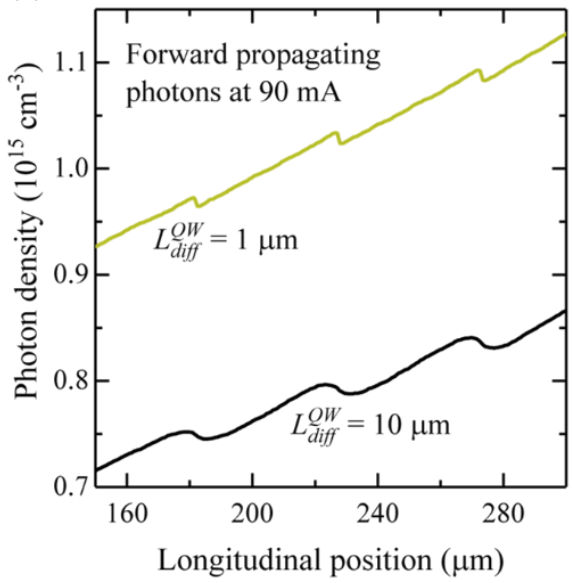

Fig. 4. Simulated $L I$ curves of an HR-coated/as-cleaved $2 \times 500 \mu \mathrm{m}^{2}$ (a) QD and (b) QW laser for various dislocation densities $\rho_{\text {dis }}$ using the parameters shown in Table I. (c) Inset of the forward propagating photon density in a QW laser versus the longitudinal position for two different diffusion lengths. 
threshold currents and, in the case of QD devices, a reduced slope efficiency [29], [30]. The carrier lifetime reduction is a well-known origin of a linearly shifted $L I$ curve towards higher currents [14], yet from the direct comparison of Figs. 4(a) and (b) it becomes obvious that the modelled QW laser suffers from a much larger threshold current increase than its QD counterpart. The key reason for this is the detrimental impact of lateral carrier diffusion, which allows diffusion-assisted BL and QW carrier loss within a radius of several micrometers around a dislocation. The influence of carrier diffusion in QD active regions, in contrast, is naturally much reduced due to rapid carrier capture into QDs, where the carriers are laterally confined and remain isolated from nearby dislocations [11]. For this reason, QD lasers require a much higher dislocation density than QWs to reduce the overall carrier density to a critical level where it limits QD carrier capture and begins to increase the laser threshold more significantly. It should be clarified that the absolute threshold current increase with dislocation density depends on the initial laser threshold without dislocation. Since many practical QW devices would have shorter cavities, which allow lower thresholds, slightly better performance than shown in Fig. 4(b) would be achieved for a $200 \mu \mathrm{m}$ long QW device, for example. The trend of with dislocation density rapidly increasing threshold currents, however, remains unchanged.

The second typical feature, the $L I$ slope reduction, is a result of two mechanisms. The first one is the reduction in local gain around dislocations, since the photon density building up while propagating along the laser cavity experiences a slight drop in these regions, as illustrated in Fig. 4(c). Hence, it can be understood intuitively that photons travelling along a laser cavity with many dislocations experience less amplification than photons in the presence of only a few dislocation-induced absorptive regions. While the absorption strength of dislocations is much more pronounced in QW lasers, as is evident from the comparison of Figs. 3(a) and (b), it should be noted that QD lasers, with their smaller modal gain, will be less able than QWs to compensate for locally reduced gain. Practically, in QW lasers this effect is overshadowed by the rapid threshold current increase, as illustrated in Fig. 5(a).

The second contribution to the slope efficiency reduction is effectively a reduced current injection efficiency due to excess dislocation-induced carrier loss in the continuum states. As shown in Fig. 5(b), the QD $L I$ slope begins to decrease substantially from $\rho_{\text {dis }} \gtrsim 10^{7} \mathrm{~cm}^{-2}$, where the BL and WL carrier densities have started to decrease to a level where sufficient carrier capture into the QDs becomes problematic. This effect is more dominant in QD lasers, which allow lasing at higher dislocation densities in the first place. A similar trend was observed in our results published in [9], yet the simulation of dislocations based solely on a macroscopically averaged carrier loss time constant led to a smaller slope decrease than observed here and in [29], for example. We believe, therefore, that the reduction in local gain as a consequence of localized nonradiative recombination could be an integral element in understanding the performance of III-V lasers grown on silicon. (a)

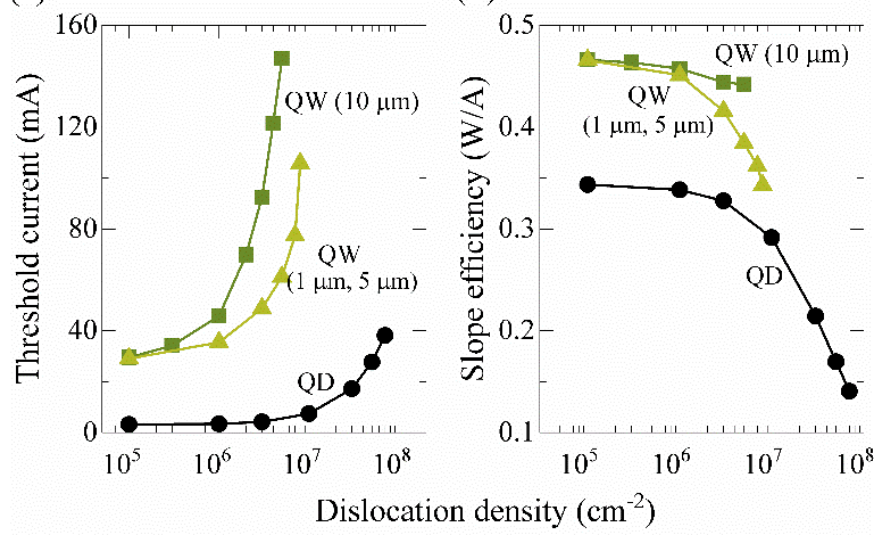

Fig. 5. (a) Threshold currents and (b) slope efficiencies extracted from Figs. 4(a) and (b) against the dislocation density. The QW laser characteristics at their original diffusion length of $10 \mu \mathrm{m}$ are additionally compared to simulations performed at $\boldsymbol{L}_{\text {diff }}^{Q W, \boldsymbol{B L}}=1 \mu \mathrm{m}, 5 \mu \mathrm{m}$.

The hypothesis that diffusion-assisted carrier loss in originally defect-free regions is a major problem for QW lasers at elevated dislocation densities, even in the theoretical absence of dislocation climb, can be confirmed by modelling QW structures at different diffusion lengths.

As shown in Fig. 5(a), the QW threshold current increase with dislocation density is substantially reduced for smaller diffusion lengths of $L_{\text {diff }}^{B L}=5 \mu \mathrm{m}$ and $L_{\text {diff }}^{Q W}=1 \mu \mathrm{m}$, similar to the behavior of the QD laser, because fewer carriers are affected by dislocation-induced carrier loss. This observation is in agreement with the performance demonstrated by $\mathrm{GaN}$ optical devices, for example, where the short minority carrier diffusion length is believed to play a key role in enabling high light emission efficiencies despite dislocation densities of up to $10^{10} \mathrm{~cm}^{-2}$ [13]. The simulations indicate, however, that the smaller thresholds come at the expense of a higher slope reduction. This is as carrier loss taking place in a more concentrated region seems to introduce a sharper drop in the local gain, which requires higher gain in dislocation-free regions, i.e. more carriers and thus a higher injection current to compensate for this.

With respect to practical applications, it should finally be noted that long-wavelength devices at $1.3 \mu \mathrm{m}$ or $1.5 \mu \mathrm{m}$ will be preferable for silicon photonics or data center singlemode applications. Since QD lasers with their lower modal gain require longer cavities, which limits the photonic chip size as well as direct modulation speeds, there might still be certain applications where QW devices could hold an advantage over their QD-based counterpart in the case that reliable, highperformance QW lasers on silicon become available.

\section{CONCLUSION}

By using a rate equation travelling-wave model with high spatial resolution, we have theoretically analyzed how individual dislocations placed along the laser cavity affect the performance of monolithic GaAs-based III-V lasers on silicon substrates. Our simulations confirm the experimentally 
observed trends of higher thresholds and reduced slope efficiencies of QD lasers on silicon, while also explaining the tendency of high threshold currents observed in monolithic QW lasers on silicon. By showing in detail how diffusion-assisted carrier loss in dislocations and the corresponding reduction in local gain influence the carrier, gain, and photon distributions in different types of active regions, our approach is capable of unveiling phenomena not observable using constant laser parameters averaged over the entire device. Two findings with particular significance for practical applications are that first, structures with a short minority carrier diffusion length show a much smaller dislocation density dependent threshold increase than QW structures with longer ones, and secondly, that increased gain in dislocation-free regions compensating for gain dips around dislocations may contribute to gain compression effects observed in directly modulated silicon-based QD lasers. In explaining the physical mechanisms still limiting the performance of III-V QW lasers on silicon, we hope to open up new pathways to be explored in order to make both monolithic III-V QD and QW lasers grown on silicon accessible to future silicon photonics applications.

\section{REFERENCES}

[1] M. Ettenberg, "Effects of dislocation density on the properties of liquid phase epitaxial GaAs," J. Appl. Phys., vol. 45, no. 2, pp. 901-906, Feb. 1974.

[2] T. L Paoli and B. W. Hakki, "cw degradation at $300^{\circ} \mathrm{K}$ of GaAs doubleheterostructure junction lasers. I. Emission spectra," J. Appl. Phys., vol. 44, no. 9, pp. 4108-4112, Sep. 1973.

[3] R. J. Roedel, A. R. Von Neida, and R. Caruso, "The effect of dislocations in $\mathrm{Ga}_{1-\mathrm{x}} \mathrm{Al}_{\mathrm{x}} \mathrm{As}$ :Si light-emitting diodes," ECS J. Solid State Sci. Technol., vol. 126, no. 4, pp. 637-341, Apr. 1979.

[4] D. Liang and J. E. Bowers, "Recent progress in lasers on silicon," Nat. Photonics, vol. 4, no. 8, pp. 511-517, Aug. 2010.

[5] L. C. Kimberling, "Recombination enhanced defect reactions," Solid State Electron., vol. 21, no. 11-12, pp. 1391-1401, Nov.-Dec. 1987.

[6] J. P. van der Ziel, R. D. Dupuis, R. A. Logan, and C. J. Pinzone, "Degradation of GaAs lasers grown by metalorganic chemical vapor deposition on Si substrates," Appl. Phys. Lett., vol. 51, no. 2, pp. 89-91, July 1987.

[7] R. B. Martins, P. Henoc, B. Akamatsu, G. Bartenlian, and M. N. Charasse, "Scanning electron microscopy investigations of the initial degradation mechanism of GaAs quantum well lasers grown on silicon substrates," $J$. Appl. Phys., vol. 66, no. 3, pp. 937-942, Aug. 1990.

[8] Z. I. Kazi, Pr. Thilakan, T. Egawa, M. Umeno, and T. Jimbo, "Realisation of GaAs/AlGaAs lasers on Si substrates using epitaxial lateral overgrowth by MOCVD," Jpn. J. Appl. Phys., vol. 40, no. 8, 99. 4903-4906, Aug. 2001.

[9] Z. Liu et al., "Origin of defect tolerance in InAs/GaAs quantum dot lasers grown on silicon," J. Lightw. Technol., published online, DOI: 10.1109/JLT.2019.2925598, 2019.

[10] A. Y. Liu, S. Srinivasan, J. Norman, A. C. Gossard, and J. E. Bowers, "Quantum dot lasers for silicon photonics," Photon. Res., vol. 3, no. 5, pp. B1-B9, Oct. 2015.

[11] D. P. Popescu, P. G. Eliseev, A. Stintz, and K. J. Malloy, "Carrier migration in structures with InAs quantum dots," J. Appl. Phys., vol. 94, no. 4, pp. 2454-2458, Aug. 2003.

[12] J. Selvidge et al., "Non-radiative recombination at dislocations in InAs quantum dots grown on silicon," Appl. Phys. Lett., vol. 115, no. 13, pp. 131102-1-131102-5, Sep. 2019.

[13] T. Sugahara et al., "Direct evidence that dislocations are non-radiative recombination centers in GaN," Jpn. J. Appl. Phys., vol. 37, no. 4A, pp. L398-L400, Apr. 1998.

[14] A. Fiore and A. Markus, "Differential gain and gain compression in quantum-dot lasers," IEEE J. Quantum Electron., vol. 43, no. 3, pp. 287297, Mar. 2007.
[15] A. Y. Liu et al., "Electrically pumped continuous-wave $1.3 \mu \mathrm{m}$ quantumdot lasers epitaxially grown on on-axis (001) GaP/Si," Opt. Express, vol. 42, no. 2, pp. 338-341, Jan. 2017.

[16] S. Chen et al., "Electrically pumped continuous-wave III-V quantum dot lasers on silicon," Nat. photonics, vol. 10, no. 5, pp. 307-312, Mar. 2016.

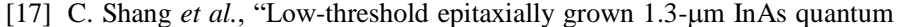
dot lasers on patterned (001) Si," IEEE J. Sel. Top. Quantum Electron., vol. 25, no. 6, pp. 1502207, Nov./Dec. 2019.

[18] A. Lee, Q. Jiang, M. Tang, A. Seeds, and H. Liu, "Continuous-wave InAs/GaAs quantum-dot laser diodes monolithically grown on Si substrate with low threshold current densities," Opt. Express, vol. 20, no. 20, pp. 22181-22187, Sep. 2012.

[19] D. Jung et al., "High efficiency low threshold current $1.3 \mu \mathrm{m}$ InAs quantum dot laser on on-axis (001) GaP/Si,"Appl. Phys. Lett., vol. 111, no. 12, pp. 122107-1-122107-4, Sep. 2017.

[20] T. H. Windhorn, G. M. Metze, B.-Y. Tsaur, and J. C. C. Fan, "AlGaAs double-heterostructure diode lasers fabricated on a monolithic $\mathrm{GaAs} / \mathrm{Si}$ substrate," Appl. Phys. Lett., vol. 45, no. 4, pp. 309-311, Aug. 1984.

[21] J.-H. Kim, G. Radhakrishnan, A. Nouhi, J. K. Liu, J. Lang, and J. Katz, "High-power $\mathrm{AlGaAs} / \mathrm{GaAs} \mathrm{DH}$ stripe laser diodes on GaAs-on-Si prepared by migration-enhanced molecular beam epitaxy," Jpn. J. Appl. Phys., vol. 28, no. 5, pp. 791-796, May 1989.

[22] M. E. Groenert et al., "Monolithic integration of room-temperature cw $\mathrm{GaAs} / \mathrm{AlGaAs}$ lasers on $\mathrm{Si}$ substrates via relaxed graded $\mathrm{GeSi}$ buffer layers," J. Appl. Phys., vol. 93, no. 1, pp. 362-367, Jan. 2003.

[23] V. Y. Aleshkin et al., "Monolithically integrated InGaAs/GaAs/AlGaAs quantum well laser grown by MOCVD on exact $\mathrm{Ge} / \mathrm{Si}(001)$ substrate," Appl. Phys. Lett., vol. 109, no. 6, pp. 06111-1-061111-5, Aug. 2016.

[24] T. C. Chong and C. G. Fonstad, "Low-threshold operation of $\mathrm{AlGaAs} / \mathrm{GaAs}$ multiple quantum well lasers grown on $\mathrm{Si}$ substrates by molecular beam epitaxy," Appl. Phys. Lett., vol. 51, no. 4, pp. 221-223, July 1987.

[25] J. P. van der Ziel, R. D. Dupuis, R. A. Logan, R. M. Mikulzak, C. J. Pinzone, and A. Savage, "Low threshold pulsed and continuous laser oscillation from $\mathrm{AlGaAs} / \mathrm{GaAs}$ double heterostructures grown by metalorganic chemical vapour deposition on Si substrates," Appl. Phys. Lett., vol. 50, no. 8, pp. 454-456, Feb. 1987.

[26] H. K. Choi and C. A. Wang, "GaAs-based diode lasers on Si with increased lifetime obtained by using strained InGaAs active layer," Appl. Phys. Lett., vol. 59, no. 21, pp. 2634-2635, Nov. 1991.

[27] M. E. Groenert, A. J. Pitera, R. J. Ram, and E. A. Fitzgerald, "Improved room-temperature continuous wave GaAs/AlGaAs and InGaAsGaAs/AlGaAs lasers fabricated on Si substrates via relaxed graded $\mathrm{Ge}_{\mathrm{x}} \mathrm{Si}_{1-\mathrm{x}}$ buffer layers," J. Vac. Sci. Technol. B., vol. 21, no. 3, pp. 10641069, May/June 2003.

[28] J. Wang et al., "Extremely low-threshold current density in InGaAs/AlGaAs quantum-well lasers on silicon," J. Lightw. Technol., vol. 33, no. 15, pp. 3136-3169, Aug. 2015.

[29] D. Jung et al., "Impact of threading dislocation density on the lifetime of InAs quantum dot lasers on Si," Appl. Phys. Lett., vol. 112, no. 15, pp. 153507-1-153507-4, Apr. 2018.

[30] J. Orchard et al., "In situ annealing enhancement of the optical properties and laser device performance of InAs quantum dots grown on $\mathrm{Si}$ substrates," Opt. Express, vol. 24, no. 6, pp. 6196-6202, Mar. 2016.

[31] D. McDonald, and R. F. O'Dowd, "Comparison of two- and three-level rate equations in the modelling of quantum-well lasers," IEEE J. Quantum Electron., vol. 31, no. 11, pp. 1927-1934, Nov. 1995

[32] K. A. Williams, M. G. Thompson, and I. H. White, "Long-wavelength monolithic mode-locked diode lasers," New J. Phys., vol. 6, no. 1, pp. 179, Nov. 2004

[33] C. Hantschmann et al., "Understanding the bandwidth limitations in monolithic $1.3 \mu \mathrm{m}$ InAs/GaAs quantum dot lasers on silicon," J. Lightw. Technol., vol. 37, no. 3, pp. 949-955, Feb. 2019.

[34] M. Gioannini, A. Sevega, and I. Montrosset, "Simulations of differential gain and linewidth enhancement factor of quantum dot semiconductor lasers," Opt. Quant. Electron., vol. 38, no. 4-6, pp. 381-394, Mar. 2006.

[35] C. L. Andre et al., "Impact of dislocations on minority carrier electron and hole lifetimes in GaAs grown on metamorphic SiGe substrates," Appl. Phys. Lett., vol. 84, no. 18, pp. 3447-3449, May 2004.

[36] J. Carroll, J. Whiteaway, and D. Plumb, "Numerical modelling for DFB lasers," in Distributed Feedback Semiconductor Lasers, London, UK: IET, 1998, pp. 209-251.

[37] S. Watanabe, Y. Kakuta, and O. Hashimoto, "Analytical study of rectangle wave propagation in electromagnet field using FDTD, CIP, and R-CIP 
methods," Microw. Opt. Technol. Lett., vol. 48, no. 10, pp. 1940-1943, Oct. 2006.

[38] K. Gündoğdu, K. C.Hall, T. F. Boggess, D. G. Deppe, and O. B. Shchekin, "Ultrafast electron capture into $p$-modulation-doped quantum dots," Appl. Phys. Lett., vol. 85, no. 20, pp. 4570-4572, Nov. 2004.

[39] K. W. Sun, A. Kechiantz, B. C. Lee, and C. P. Lee, "Ultrafast carrier capture and relaxation in modulation-doped InAs quantum dots," Appl. Phys. Lett., vol. 88, no. 16, pp. 163117-1-163117-2, Apr. 2006.

[40] K. Löhnert and E. Kubalek, "The cathodoluminescence contrast formation of localized non-radiative defects in semiconductors," Phys. Stat. Sol. A, vol. 83, no, 1, pp. 307-314, May 1987.

[41] W. R. Harding, I. D. Blenkinskop, and D. R. Wight, "Dislocation-limited minority-carrier lifetime in $n$-type GaP," Electron. Lett., vol. 12, no. 19, pp. 503-504, Sep. 1976.

[42] W. R. Harding, I. D. Blenkinsop, and D. R. Wright, "Dislocation-limited minority-carrier lifetime in $n$-type GaP," Electron. Lett., vol. 12, no. 19, pp. 503-504, Sep. 1976.

[43] H. Y. Liu et al., "Improved performance of $1.3 \mu \mathrm{m}$ multilayer InAs quantum-dot lasers using a high-growth-temperature GaAs spacer layer," Appl. Phys. Lett., vol. 85, no. 5, pp. 704-706, Aug. 2004.

[44] A. Y. Liu et al., „MBE growth of P-doped $1.3 \mu \mathrm{m}$ quantum dot lasers on silicon," J. Vac. Sci. Technol. B, vol. 32, no. 2, pp. 02C108-1-02C108-4, Mar./Apr. 2014.

[45] F. Grillot, B. Dagens, J.-G. Provost, H. Su, and L. F. Lester, "Gain compression and above-threshold linewidth enhancement factor in $1.3-\mu \mathrm{m}$ InAs-GaAs quantum-dot lasers," IEEE J. Sel. Quantum Electron., vol. 44, no. 10 , pp. 946-951, Oct. 2008.

[46] J. Duan et al., "Dynamic and nonlinear properties of epitaxial quantum dot lasers on silicon for isolator-free integration," Photonics Res., vol. 7, no. 11, pp. 1222-1228, Nov. 2019.

[47] C. Hantschmann et al., "Dynamic properties of monolithic $1.3 \mu \mathrm{m}$ InAs/GaAs quantum dot lasers on silicon," in Proc. IEEE Int. Semicond. Laser Conf., Santa Fe, NM, USA, 2018, pp. 139-140. 